\title{
Phenol Derivatives as Coupling Partners with Alkylsilicates in Photoredox/Nickel Dual Catalysis
}

\author{
Niki R. Patel and Gary A. Molander* \\ Roy and Diana Vagelos Laboratories, Department of Chemistry, University of Pennsylvania, 231 South 34th Street, Philadelphia, \\ Pennsylvania 19104-6323, United States
}

\section{Supporting Information}

ABSTRACT: Photoredox/nickel dual catalysis via single electron transmetalation allows coupling of $\mathrm{C}_{\mathrm{sp}}{ }^{3}-\mathrm{C}_{\mathrm{sp}}{ }^{2}$ hybridized centers under mild conditions. A procedure for the coupling of electrondeficient aryl triflates, -tosylates, and -mesylates with alkylbis(catecholato)silicates is presented. This method represents the first example of the use of phenol derivatives as electrophilic coupling partners in photoredox/nickel dual catalysis.

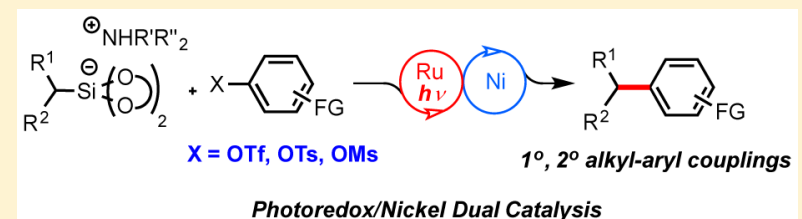

Photoredox/Nickel Dual Catalysis
$\mathrm{T}$ ransition-metal-catalyzed cross-couplings have become the foundation by which synthetic chemists generate $\mathrm{C}-\mathrm{C}$ and $\mathrm{C}-$ heteroatom bonds. ${ }^{1}$ Despite the tremendous impact of such methods, $\mathrm{C}-\mathrm{C}$ bond-forming reactions using transition metal catalysts are often restricted to the construction of $\mathrm{C}_{\mathrm{sp}}{ }^{2}-\mathrm{C}_{\mathrm{sp}}{ }^{2}$ bonds, with extension to $\mathrm{C}_{\mathrm{sp}}{ }^{3}-\mathrm{C}_{\mathrm{sp}}{ }^{2}$ cross-couplings proving challenging. ${ }^{2}$ Recent developments, however, have enabled $\mathrm{C}_{\mathrm{sp}}{ }^{3}-\mathrm{C}_{\mathrm{sp}}{ }^{2}$ bonds to be forged with ease via photoredox/nickel dual catalysis. ${ }^{3}$ In particular, we and others have recently highlighted the success of alkylbis(catecholato)silicates as efficient radical precursors in dual catalysis. ${ }^{4}$ Alkylbis(catecholato)silicates undergo facile SET oxidation via reductive quenching of a variety of photocatalysts that results in $\mathrm{C}-\mathrm{Si}$ bond homolysis to produce $\mathrm{C}_{\mathrm{sp}}{ }^{3}$-centered radicals. ${ }^{5}$ Because of their low oxidation potentials, the use of $\left[\mathrm{Ru}(\mathrm{bpy})_{3}\right]\left(\mathrm{PF}_{6}\right)_{2}$ as a photocatalyst, which is both commercially available or simply made in-house, is feasible. ${ }^{6}$

To date, $\mathrm{C}_{\mathrm{sp}}{ }^{3}-\mathrm{C}_{\mathrm{sp}}{ }^{2}$ cross-couplings via photoredox/nickel dual catalysis have been carried out using only aryl/alkenyl halide starting materials. ${ }^{3 a, b, 4,7}$ However, phenol derivatives, or "pseudo-halides", can often be used as replacements for aryl halides in traditional transition-metal-catalyzed cross-couplings. ${ }^{8}$ Despite their use in these reactions, the ability to cross-couple aryl sulfonate esters with alkyl nucleophiles is limited, analogous to their halide counterparts. ${ }^{9}$ Because they are derived from different feedstocks than aryl halides, aryl triflates, -tosylates, and -mesylates allow access to cross-coupled substructures that may be complementary to those derived from the aryl halides alone. In addition, the ease of synthesis of aryl sulfonates from the corresponding phenols contrasts with that of the corresponding halides, which requires halogenation of arenes or aryl diazonium salts.

In an effort to expand the scope of alkylsilicate photoredox/ $\mathrm{Ni}$ dual catalytic cross-coupling with suitable electrophiles, the compatibility of aryl sulfonates was assessed. Because they possess a similar propensity toward oxidative addition as that of the corresponding aryl iodides and bromides, ${ }^{10}$ aryl triflates and other phenol derivatives can be thought of as suitable replacements in the proposed catalytic cycle (Figure 1). ${ }^{11}$

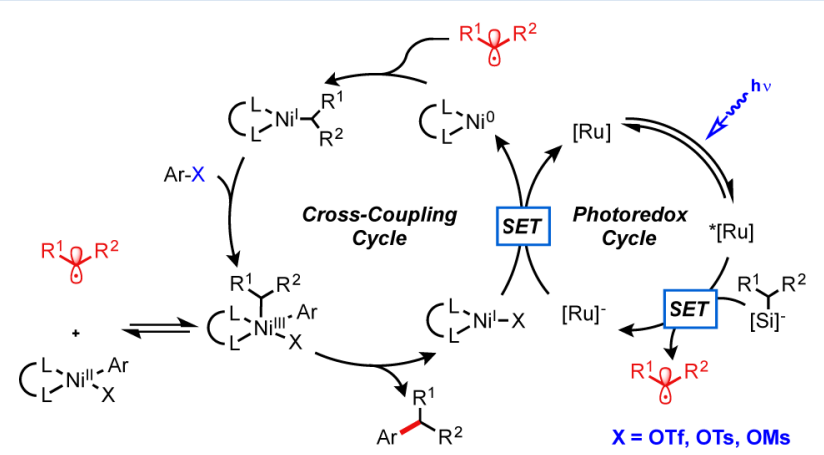

Figure 1. Proposed catalytic cycle for photoredox/Ni dual catalysis with alkylsilicates and phenol derivatives.

To assess the viability of aryl sulfonates in photoredox/ $\mathrm{Ni}$ dual catalysis, a preliminary screening revealed an $87 \%$ conversion of 4-acetylphenyl triflate (2a) when the reaction was conducted in the presence of 1a under our previously established conditions $\left\{2 \mathrm{~mol} \%\left[\mathrm{Ru}(\mathrm{bpy})_{3}\right]\left(\mathrm{PF}_{6}\right)_{2}, 5 \mathrm{~mol} \%\right.$ $\mathrm{NiCl}_{2}$ (dme), $5 \mathrm{~mol} \%$ dtbbpy in DMF (0.1 M), $26 \mathrm{~W}$ compact fluorescence lamp (CFL) or blue LEDs $\}$ after $24 \mathrm{~h}^{4 \mathrm{~b}, \mathrm{c}, 12}$ Further optimization of this reaction allowed full consumption of triflate $2 \mathrm{a}$ when increasing $\mathrm{NiCl}_{2}(\mathrm{dme})$ and dtbbpy loading to $10 \mathrm{~mol} \%$ (see Supporting Information), which gave 3a in $95 \%$ yield on a $0.5 \mathrm{mmol}$ scale after $36 \mathrm{~h}^{13}$ Using these conditions, the full scope of this reaction was explored using $\mathbf{1 a}$ as the silicate coupling partner (Table 1 ). In addition to triflate 2a, silicate 1a coupled successfully to both 1- and 2-naphthyl triflates in $94 \%$ (3c) and 50\% (3d) yields, respectively. Minimal

Special Issue: Photocatalysis

Received: April 11, 2016

Published: June 3, 2016 
Table 1. Scope of Aryl Triflates in Photoredox/Ni CrossCoupling ${ }^{a, b}$

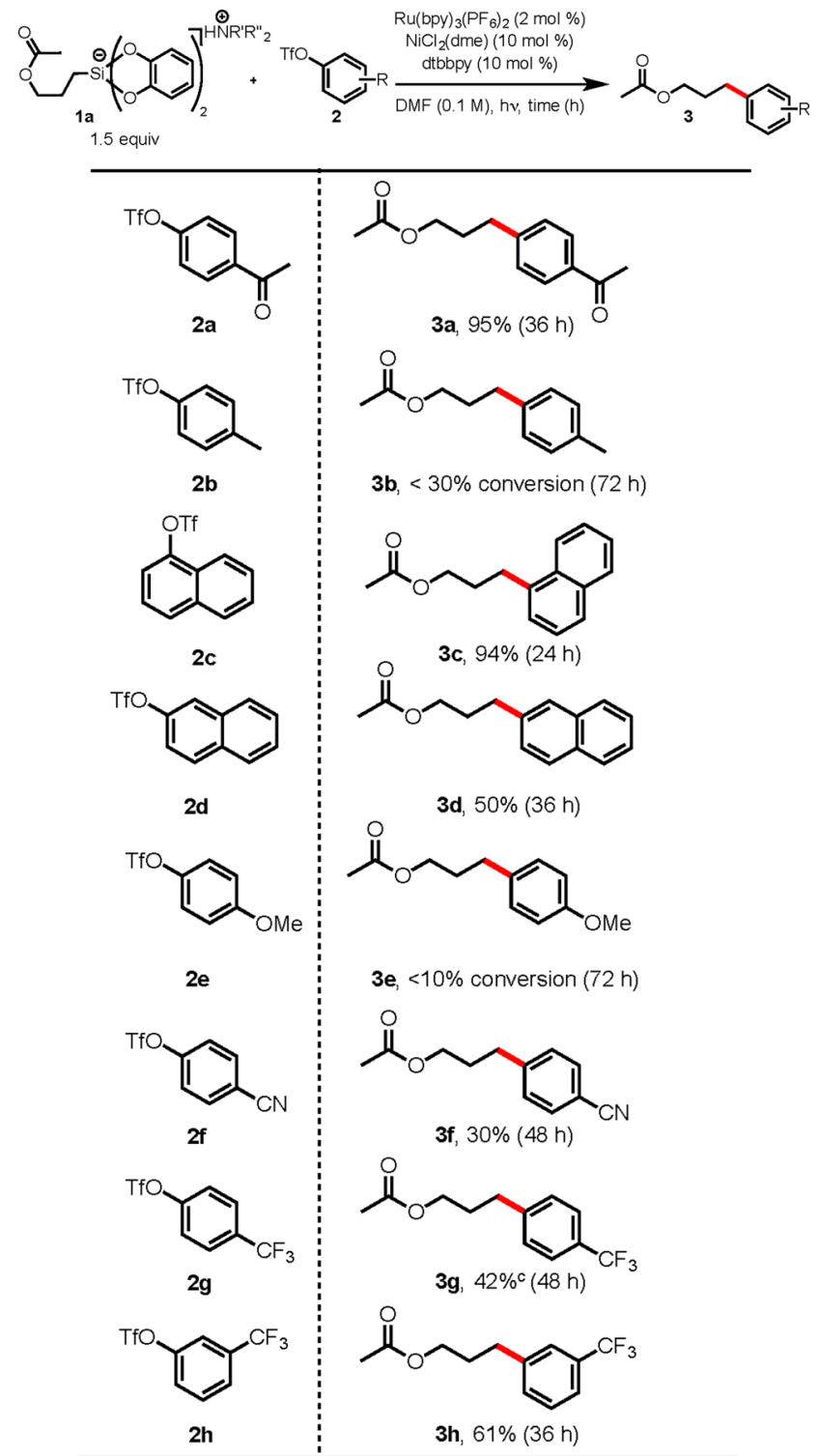

${ }^{a} \mathrm{R}^{\prime}=\mathrm{R}^{\prime \prime}=$ Et or $\mathrm{R}^{\prime}=\mathrm{H}, \mathrm{R}^{\prime \prime}=i$-Pr. ${ }^{b}$ Conversion determined by HPLC. ${ }^{c}$ Inseparable aryl dimer present in ${ }^{1} \mathrm{H}$ and ${ }^{13} \mathrm{C}$ NMRs.

conversion was observed in the case of $3 \mathbf{e}$, possessing an electron-donating methoxy group at the para position of the arene. Additionally, although this reaction has been shown to work well with electron-deficient aryl triflates, low yields were obtained when attempting to couple silicate 1a with cyano- and trifluoromethyl-containing aryl triflates, $\mathbf{2 f}$ and $\mathbf{2 g}$, respectively. Although unreacted starting material remained in the reaction of $\mathbf{2 f}$, complete conversion of triflate was observed in the case of $2 \mathrm{~g}$, in which the reaction yielded a mixture of cross-coupled product $3 \mathbf{g}$ as well as aryl dimer from the triflate starting material. It should be noted that dimerization was not observed in reactions other than those containing trifluoromethyl functional groups. Interestingly, this reaction proved viable for meta-substituted aryl triflate derivative $\mathbf{2 h}$, providing crosscoupled product $3 \mathbf{h}$ in good yield.

The scope of alkylbis(catecholato)silicates in this reaction was next examined (Table 2). ${ }^{14}$ Moderate to excellent yields were obtained for a variety of primary and secondary
Table 2. Scope of Alkylbis(catecholato)silicates in Photoredox/Ni Cross-Coupling ${ }^{a}$

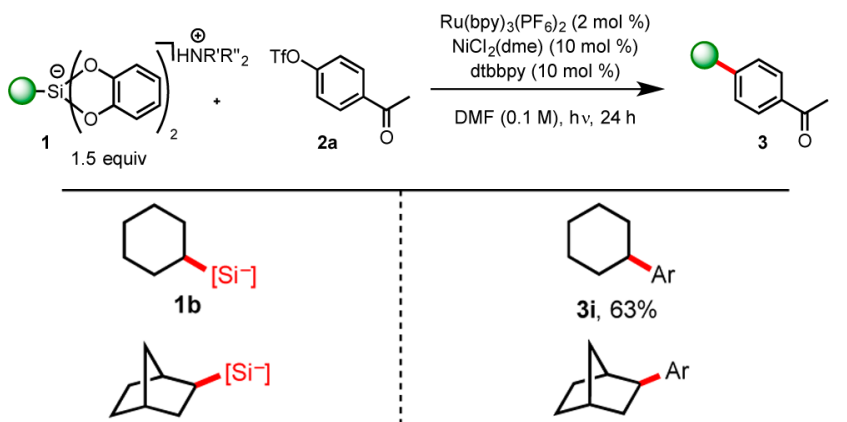

$( \pm)-3 \mathbf{j}, 92 \%$

$( \pm)-1 c$<smiles>[SiH3]Cc1ccccc1</smiles>

1d

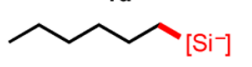

$1 e$

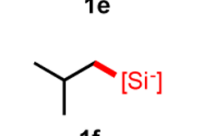

$1 f$

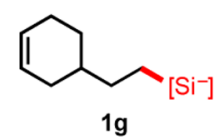

$\mathrm{MeO}_{\left[\mathrm{Si}^{-}\right]}$

$1 \mathrm{~h}$

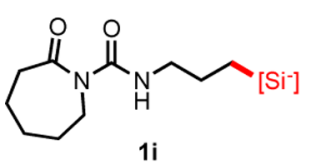

A $A r$

3 k, $33 \%^{b}$<smiles>CCCCCCC[Te]</smiles>

31, $74 \%$<smiles>CC(C)C[Al]</smiles>

$3 \mathrm{~m}, 78 \%,{ }^{\mathrm{c}} 82 \%{ }^{\mathrm{d}}$

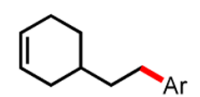

3n, $55 \%$<smiles>COCCC[Al]</smiles>

3o, $75 \%$

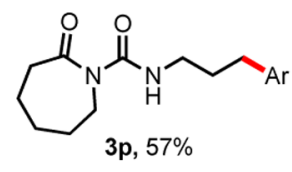

${ }^{a} \mathrm{R}^{\prime}=\mathrm{R}^{\prime \prime}=$ Et or $\mathrm{R}^{\prime}=\mathrm{H}, \mathrm{R}^{\prime \prime}=i$-Pr. ${ }^{b} \mathrm{NMR}$ yield. ${ }^{c}$ Reaction provided comparable yield when no measures were taken to remove oxygen. ${ }^{d}$ Reaction with $3 \mathrm{~m}$ run on gram scale using $5 \mathrm{~mol} \%\left[\mathrm{NiCl}_{2}(\mathrm{dme})\right]$ and dtbbpy with white LEDs.

alkylsilicates when reacted with triflate 2 a. Secondary silicates $\mathbf{1 b}$ and $\mathbf{1 c}$ were amenable to cross-coupling, generating the expected products in $63 \%$ and $92 \%$ yields, respectively. Additionally, simple primary silicates were amenable to crosscoupling. Hexyl (1e) and isobutyl (1f) silicate derivatives thus provided cross-coupled products in $74 \%$ and $78 \%$ yields, respectively. Silicate 1 ff performed well on gram scale, generating cross-coupled product in $82 \%$ yield using $5 \mathrm{~mol} \%$ $\mathrm{NiCl}_{2}$ (dme) and dtbbpy. Furthermore, the conditions of this reaction successfully allowed alkyl chains containing both alkenyl and methoxy functional groups ( $\mathbf{n}$ n and $\mathbf{3 o}$, respectively), as well as a seven-membered ring lactam/urea (1i) to be installed with ease. Importantly, the reaction could be carried out in the presence of oxygen, and thus no degassing of solvents was necessary.

Although aryl triflates are often prone to hydrolysis under basic conditions, aryl tosylates and -mesylates exhibit better stability and are easier to handle and store. Given the observed reactivity utilizing aryl triflates as electrophilic coupling partners in photoredox/nickel dual catalysis in the presence of alkylbis(catecholato)silicates, the scope of this reaction using aryl tosylates was explored (Table 3). In the event, acetyl- 
Table 3. Cross-Coupling of Aryl Tosylates with Alkylbis(catecholato)silicates in Photoredox/Ni CrossCoupling ${ }^{a}$
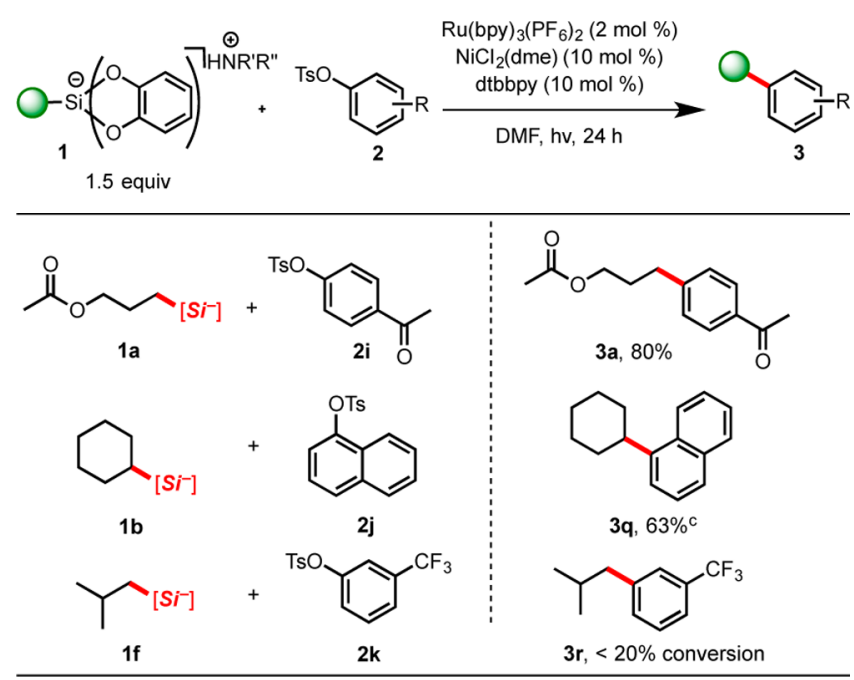

${ }^{a} \mathrm{R}^{\prime}=\mathrm{R}^{\prime \prime}=$ Et or $\mathrm{R}^{\prime}=\mathrm{H}, \mathrm{R}^{\prime \prime}=i$-Pr. ${ }^{b}$ Conversion determined by HPLC. ${ }^{c}$ Inseparable cyclohexyl dimer impurity present in ${ }^{1} \mathrm{H}$ and ${ }^{13} \mathrm{C}$ NMRs

containing aryl tosylate $2 \mathbf{i}$ showed similar reactivity to its analogous triflate derivative, furnishing $3 \mathbf{a}$ in $80 \%$ yield. In addition, 1-naphthyl tosylate $\mathbf{2 j}$ proved a sufficient coupling partner with secondary silicate $\mathbf{1 b}$. Despite the success of metasubstituted triflate derivative $\mathbf{2 h}$; however, tosylate $\mathbf{2 k}$ did not prove to be an adequate coupling partner.

Along with triflates and tosylates, mesylates were found to be competent electrophilic coupling partners in this transformation. Coupling products $3 \mathrm{a}$ and $3 \mathrm{~s}$ were produced in $54 \%$ and $72 \%$ yields, respectively, using aryl methansulfonates $\mathbf{2 l}$ and $\mathbf{2 m}$ (eqs 1 and 2). Unfortunately, sulfamate and carbamate derivatives proved to be unsuccessful coupling partners under this set of conditions.

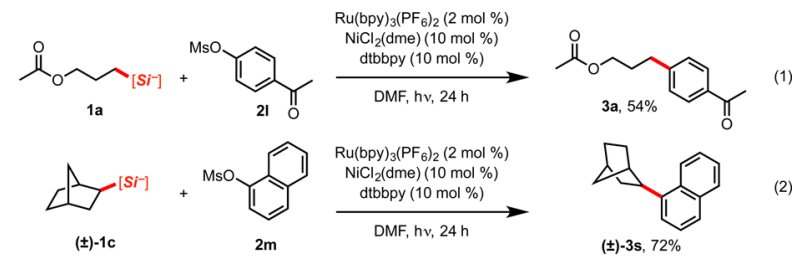

In addition to the substrates described above, other alkylsilicates and aryl triflates were attempted in the crosscoupling (Table 4). In general, we observed reduced yields using electron-rich substrates, likely because of the additive effects of this class of substrate with the diminished rate of oxidative addition into aryl sulfonate esters compared to their analogous halide counterparts. Despite the previous success of nitrogen-containing silicates with aryl- and alkenyl halides, ${ }^{4 \mathrm{~b}, \mathrm{c}} \mathbf{1 j}$ and $\mathbf{~ k ~ p r o v e d ~ t o ~ b e ~ p o o r ~ n u c l e o p h i l i c ~ c o u p l i n g ~ p a r t n e r s ~ f o r ~}$ this transformation when reacted with $2 \mathrm{a}$ and $\mathbf{2 c}$. In addition, triflates $2 n-2 s$ proved to be unsuccessful when reacted with silicate 1a, generating little to no cross-coupled product after 48 h.

In view of the previously reported relative reactivity of aryl halides in this reaction, ${ }^{4 \mathrm{~b}}$ the competition between aryl triflates and -bromides in the cross-coupling was next assessed. When
Table 4. Unsuccessful Cross-Coupling Partners in Photoredox/Ni Cross-Coupling ${ }^{a}$

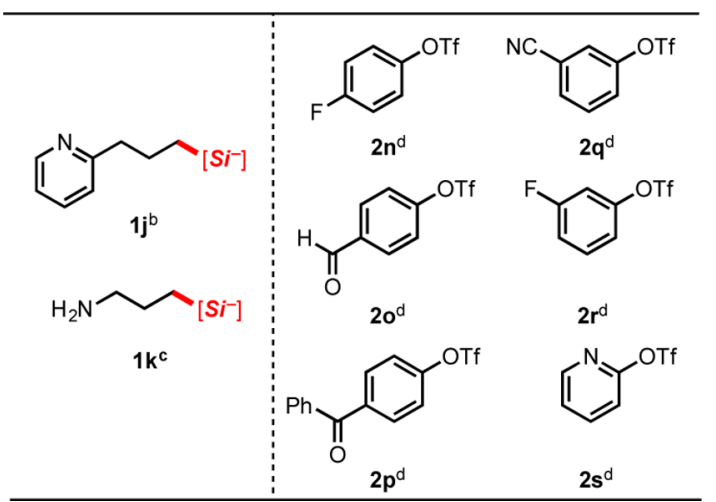

${ }^{a}$ Reacted using $2 \mathrm{~mol} \%\left[\mathrm{Ru}(\mathrm{bpy})_{3}\right]\left(\mathrm{PF}_{6}\right), 10 \mathrm{~mol} \%\left[\mathrm{NiCl}_{2}(\mathrm{dme})\right]$ and $10 \mathrm{~mol} \% \mathrm{dttbpy}$ in DMF $(0.1 \mathrm{M}) .{ }^{b}$ Reacted with $2 \mathrm{a} .{ }^{c}$ Reacted with 2 c. ${ }^{d}$ Reacted with silicate $\mathbf{1 a}$.

subjecting $2 t$ to the conditions of the cross-coupling reaction in the presence of silicate 1a (eq 3 ), the reaction occurred solely

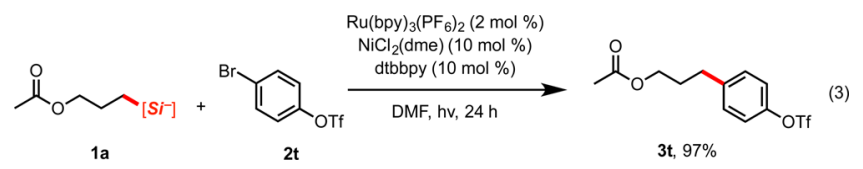

at the halide, leaving the triflate virtually unreacted (3t). This suggests that the faster rate of oxidative addition of the aryl halide onto $\mathrm{Ni}(\mathrm{I})$ is critical in determining reactivity. Reactivity solely at the halide could allow selective photoredox/ $\mathrm{Ni}$ catalyzed cross-coupling at the bromide followed by subsequent reactivity at the triflate via photoredox/Ni dual catalysis or by means of traditional Pd- or Ni-catalyzed cross-coupling.

In summary, electron-deficient aryl sulfonates can be readily cross-coupled with ammonium alkylbis(catecholato)silicates via photoredox/nickel dual catalysis-processes that proceed via single electron transmetalation under mild conditions. The ease of SET oxidation of alkylbis(catecholato)silicates to produce $\mathrm{C}_{\mathrm{sp}}{ }^{3}$ centered radicals permits the use of a readily available photocatalyst. The compatibility of phenol derivatives toward oxidative addition of nickel centers allows the expansion of aryl-alkyl couplings via photoredox-nickel dual catalysis to include sulfonate ester based electrophiles. Electron-deficient aryl triflates, -tosylates, and -mesylates have been shown to be successful electrophilic coupling partners in the described crosscoupling reaction. This method represents the first example of the use of phenol derivatives as electrophilic coupling partners in photoredox/nickel dual catalysis.

\section{EXPERIMENTAL SECTION}

General Procedure for Photoredox Cross-Coupling. 3-(4Acetylphenyl)propyl Acetate (3a). ${ }^{15}$ To a 2 dram, clear glass vial equipped with a Teflon-coated magnetic stir bar were added 4,4'-ditert-butyl-2,2'-bipyridine $(13.4 \mathrm{mg}, 0.050 \mathrm{mmol})$ and $\left[\mathrm{NiCl}_{2}(\mathrm{dme})\right]$ $(11 \mathrm{mg}, 0.050 \mathrm{mmol})$. The vial was sealed and evacuated three times via an inlet needle and purged with argon. Once purged, $1.5 \mathrm{~mL}$ of THF was introduced. The resulting suspension was heated briefly with a heat gun until the nickel and ligand were fully solubilized, yielding a pale green solution. Solvents were then evaporated in vacuo to give a fine coating of the ligated nickel complex. Once dry, a phenol derivative (134 mg, $0.5 \mathrm{mmol}, 1.0$ equiv) (liquid phenol derivatives were added with solvent), alkylsilicate ( $335 \mathrm{mg}, 0.75 \mathrm{mmol}, 1.5$ equiv), and $\left[\mathrm{Ru}(\mathrm{bpy})_{3}\right]_{2} \mathrm{PF}_{6}(8.6 \mathrm{mg}, 0.01 \mathrm{mmol})$ were added in succession. 
Under an inert atmosphere, DMF $(5 \mathrm{~mL})$ was introduced. The cap was sealed with Parafilm, and the solution was irradiated in front of a $26 \mathrm{~W}$ CFL bulb or blue LEDs. The temperature of the reaction was maintained at approximately $27^{\circ} \mathrm{C}$ via a fan. The solution was stirred vigorously while being irradiated. The reaction progress was monitored by HPLC or GC/MS. Once judged to be complete, the solution was transferred to a separatory funnel and diluted with deionized $\mathrm{H}_{2} \mathrm{O}(\sim 20 \mathrm{~mL})$ and $\mathrm{Et}_{2} \mathrm{O}(\sim 20 \mathrm{~mL})$. The layers were separated, and the aqueous layer was extracted with $\mathrm{Et}_{2} \mathrm{O}(3 \times \sim 20$ $\mathrm{mL})$. The combined organic layers were washed with $1 \mathrm{M} \mathrm{NaOH}$ $(\sim 30 \mathrm{~mL}), 1 \mathrm{M} \mathrm{HCl}(\sim 30 \mathrm{~mL})$, and brine $(\sim 50 \mathrm{~mL})$. The organic layer was dried $\left(\mathrm{MgSO}_{4}\right)$, and the solvent was removed in vacuo by rotary evaporation. Further purification was accomplished via column chromatography, eluting with hexane/EtOAc to give the desired compound as a light yellow oil (102 mg, 95\%). ${ }^{1} \mathrm{H}$ NMR $\left(\mathrm{CDCl}_{3}, 500\right.$ $\mathrm{MHz}): \delta 7.89(\mathrm{~d}, J=8.3 \mathrm{~Hz}, 2 \mathrm{H}), 7.28(\mathrm{~d}, J=8.3 \mathrm{~Hz}, 2 \mathrm{H}), 4.09(\mathrm{t}, J=$ $6.5 \mathrm{~Hz}, 2 \mathrm{H}), 2.75(\mathrm{t}, J=7.6 \mathrm{~Hz}, 2 \mathrm{H}), 2.59(\mathrm{~s}, 3 \mathrm{H}), 2.05(\mathrm{~s}, 3 \mathrm{H}), 2.02-$ $1.93(\mathrm{~m}, 2 \mathrm{H}) .{ }^{13} \mathrm{C}\left\{{ }^{1} \mathrm{H}\right\}$ NMR $\left(\mathrm{CDCl}_{3}, 125 \mathrm{MHz}\right): \delta 197.8,171.2$, 147.1, 135.4, 128.7, 128.7, 63.7, 32.4, 29.9, 26.7, 21.0.

3-(Naphthalen-1-yl)propyl Acetate (3c). ${ }^{4 b}$ Obtained as a colorless oil $(104 \mathrm{mg}, 94 \%) .{ }^{1} \mathrm{H}$ NMR $\left(\mathrm{CDCl}_{3}, 500 \mathrm{MHz}\right): \delta 8.02$ (d, $J=8.1$ $\mathrm{Hz}, 1 \mathrm{H}), 7.85(\mathrm{~d}, J=7.9 \mathrm{~Hz}, 1 \mathrm{H}), 7.72(\mathrm{~d}, J=7.9 \mathrm{~Hz}, 1 \mathrm{H}), 7.55-7.44$ $(\mathrm{m}, 2 \mathrm{H}), 7.39(\mathrm{t}, J=7.3 \mathrm{~Hz}, 1 \mathrm{H}), 7.32(\mathrm{~d}, J=7.0 \mathrm{~Hz}, 1 \mathrm{H}), 4.16(\mathrm{t}, J=$ $6.5 \mathrm{~Hz}, 2 \mathrm{H}), 3.15(\mathrm{t}, J=7.7 \mathrm{~Hz}, 2 \mathrm{H}), 2.15-2.03(\mathrm{~m}, 5 \mathrm{H}) .{ }^{13} \mathrm{C}\left\{{ }^{1} \mathrm{H}\right\}$ NMR $\left(\mathrm{CDCl}_{3}, 125 \mathrm{MHz}\right): \delta 171.2,137.3,134.0,131.9,128.9,127.0$, 126.1, 126.0, 125.6, 125.6, 123.7, 64.2, 29.6, 29.4, 21.1.

3-(Naphthalen-2-yl)propyl Acetate (3d). ${ }^{16}$ Obtained as a colorless oil $(57 \mathrm{mg}, 50 \%) .{ }^{1} \mathrm{H} \mathrm{NMR}\left(\mathrm{CDCl}_{3}, 500 \mathrm{MHz}\right): \delta 7.83-7.74(\mathrm{~m}, 3 \mathrm{H})$, $7.62(\mathrm{~s}, 1 \mathrm{H}), 7.48-7.38(\mathrm{~m}, 2 \mathrm{H}), 7.32(\mathrm{dd}, J=8.4,1.8 \mathrm{~Hz}, 1 \mathrm{H}), 4.13$ $(\mathrm{t}, J=6.6 \mathrm{~Hz}, 2 \mathrm{H}), 2.85(\mathrm{t}, J=7.6 \mathrm{~Hz}, 2 \mathrm{H}), 2.09-2.01(\mathrm{~m}, 5 \mathrm{H}) .{ }^{13} \mathrm{C}$ $\left\{{ }^{1} \mathrm{H}\right\}$ NMR $\left(\mathrm{CDCl}_{3}, 125 \mathrm{MHz}\right): \delta 171.2,138.8,133.7,132.2,128.1$, 127.7, 127.5, 127.2, 126.6, 126.1, 125.3, 64.0, 32.5, 30.2, 21.1.

3-(4-Cyanophenyl)propyl Acetate (3f). Obtained as a colorless oil (31 mg, 30\%). ${ }^{1} \mathrm{H}$ NMR $\left(\mathrm{CDCl}_{3}, 500 \mathrm{MHz}\right): \delta 7.58(\mathrm{~d}, J=7.3 \mathrm{~Hz}$, $2 \mathrm{H}), 7.27(\mathrm{~d}, J=7.7 \mathrm{~Hz}, 2 \mathrm{H}), 4.09(\mathrm{t}, J=6.2 \mathrm{~Hz}, 2 \mathrm{H}), 2.75(\mathrm{t}, J=7.7$ $\mathrm{Hz}, 2 \mathrm{H}), 2.04(\mathrm{~s}, 3 \mathrm{H}), 2.04-1.93(\mathrm{~m}, 2 \mathrm{H}) .{ }^{13} \mathrm{C}\left\{{ }^{1} \mathrm{H}\right\} \operatorname{NMR}\left(\mathrm{CDCl}_{3}\right.$, $125 \mathrm{MHz}): \delta 171.3,146.9,132.4,129.3,119.0,110.2,63.5,32.5,29.8$, 21.0. IR (neat): $\nu=2957,2227,1734,1608,1505,1453,1415,1387$, $1366,1234,1178,1038,915,878,846,815,733,633,606,559 \mathrm{~cm}^{-1}$. HRMS (ESI): $m / z$ calcd for $\mathrm{C}_{12} \mathrm{H}_{13} \mathrm{NO}_{2} \mathrm{Na}[\mathrm{M}+\mathrm{Na}]^{+} 226.0844$, found 226.0834 .

3-(4-(Trifluoromethyl)phenyl)propyl Acetate (3g). ${ }^{4 e}$ Obtained as a colorless oil $(61 \mathrm{mg}, 42 \%) .{ }^{1} \mathrm{H}$ NMR $\left(\mathrm{CDCl}_{3}, 500 \mathrm{MHz}\right): \delta 7.56(\mathrm{~d}, J$ $=8.0 \mathrm{~Hz}, 2 \mathrm{H}), 7.31(\mathrm{~d}, J=7.9 \mathrm{~Hz}, 2 \mathrm{H}), 4.10(\mathrm{t}, J=6.5 \mathrm{~Hz}, 2 \mathrm{H}), 2.76$ $(\mathrm{t}, J=7.8 \mathrm{~Hz}, 2 \mathrm{H}), 2.06(\mathrm{~s}, 3 \mathrm{H}), 2.03-1.95(\mathrm{~m}, 2 \mathrm{H}) .{ }^{13} \mathrm{C}\left\{{ }^{1} \mathrm{H}\right\} \mathrm{NMR}$ $\left(\mathrm{CDCl}_{3}, 125 \mathrm{MHz}\right): \delta 171.2,145.4,128.8,128.6(\mathrm{q}, J=32.3 \mathrm{~Hz})$, $125.5(\mathrm{q}, J=3.8 \mathrm{~Hz}), 122.3(\mathrm{q}, J=271.9 \mathrm{~Hz}), 63.7,32.2,30.0,21.1$.

3-(3-(Trifluoromethyl)phenyl)propyl Acetate (3h). Obtained as a colorless oil (103 mg, 84\%). ${ }^{1} \mathrm{H}$ NMR $\left(\mathrm{CDCl}_{3}, 500 \mathrm{MHz}\right): \delta 7.49-$ $7.34(\mathrm{~m}, 4 \mathrm{H}), 4.10(\mathrm{t}, J=6.5 \mathrm{~Hz}, 2 \mathrm{H}), 2.75(\mathrm{t}, J=7.5 \mathrm{~Hz}, 2 \mathrm{H}), 2.05$ (s, 3H), 2.03-1.93 (m, 2H). ${ }^{13} \mathrm{C}\{1 \mathrm{H}\}$ NMR $\left(\mathrm{CDCl}_{3}, 125 \mathrm{MHz}\right): \delta$ $171.2,142.2,131.9,129.0,130.9(\mathrm{q}, J=32.0 \mathrm{~Hz}), 125.2(\mathrm{q}, J=3.7$ $\mathrm{Hz}), 124.3(\mathrm{q}, J=272.2 \mathrm{~Hz}), 123.1(\mathrm{q}, J=3.9 \mathrm{~Hz}), 63.7,32.2,30.1$, 21.0. ${ }^{19} \mathrm{~F} \mathrm{NMR}\left(\mathrm{CDCl}_{3}, 470 \mathrm{MHz}\right):-62.6$. IR (neat): $\nu=1737,1450$, 1388, 1367, 1328, 1236, 1199, 1161, 1120, 1072, 1039, 1002, 951, 904, 799, 733, 702, 661, 631, $606 \mathrm{~cm}^{-1}$. HRMS (ESI): $\mathrm{m} / z$ calcd for $\mathrm{C}_{12} \mathrm{H}_{13} \mathrm{~F}_{3} \mathrm{O}_{2} \mathrm{Na}[\mathrm{M}+\mathrm{Na}]^{+}$269.0765, found 269.0754 .

1-(4-Cyclohexylphenyl)ethanone (3i). ${ }^{17}$ Obtained as a white solid (63 mg, 63\%); mp, 57-58 ${ }^{\circ} \mathrm{C} .{ }^{1} \mathrm{H}$ NMR $\left(\mathrm{CDCl}_{3}, 500 \mathrm{MHz}\right): \delta 7.89$ $(\mathrm{d}, J=8.3 \mathrm{~Hz}, 2 \mathrm{H}), 7.29(\mathrm{~d}, J=8.2 \mathrm{~Hz}, 2 \mathrm{H}), 2.58(\mathrm{~m}, 4 \mathrm{H}), 1.92-1.81$ (m, 4H), 1.80-1.72 (m, 1H), 1.50-1.34 (m, 4H), 1.33-1.18 (m, 1H). ${ }^{13} \mathrm{C}\left\{{ }^{1} \mathrm{H}\right\}$ NMR $\left(\mathrm{CDCl}_{3}, 125 \mathrm{MHz}\right): \delta 197.9,153.8,135.2,128.6$, 127.1, 44.8, 34.2, 26.8, 26.6, 26.1.

(士)-1-(4-(Bicyclo[2.2.1] heptan-2-yl)phenyl)ethanone (3j). Obtained as a yellow oil $(92 \mathrm{mg}, 92 \%) .{ }^{1} \mathrm{H} \mathrm{NMR}\left(\mathrm{CDCl}_{3}, 500 \mathrm{MHz}\right)$ : $\delta 7.87(\mathrm{~d}, J=8.5 \mathrm{~Hz}, 2 \mathrm{H}), 7.30(\mathrm{~d}, J=8.3 \mathrm{~Hz}, 2 \mathrm{H}), 2.83-2.75(\mathrm{~m}$, $1 \mathrm{H}), 2.57(\mathrm{~s}, 3 \mathrm{H}), 2.42-2.34(\mathrm{~m}, 2 \mathrm{H}), 1.84-1.70(\mathrm{~m}, 1 \mathrm{H}), 1.69-1.48$ $(\mathrm{m}, 3 \mathrm{H}), 1.41-1.34(\mathrm{~m}, 1 \mathrm{H}), 1.33-1.18(\mathrm{~m}, 3 \mathrm{H}) .{ }^{13} \mathrm{C}\left\{{ }^{1} \mathrm{H}\right\} \mathrm{NMR}$ $\left(\mathrm{CDCl}_{3}, 125 \mathrm{MHz}\right): \delta 197.9,153.5,134.8,128.5,127.3,47.6,42.8$, 39.2, 37.0, 36.3, 30.7, 28.9, 26.6. IR (neat): $\nu=2949,2869,1679$,
$1604,1568,1454,1411,1357,1308,1267,1212,1185,1139,1014$ $955,848,822,605,591,570 \mathrm{~cm}^{-1}$. HRMS (ESI): $\mathrm{m} / z$ calcd for $\mathrm{C}_{15} \mathrm{H}_{19} \mathrm{O}[\mathrm{M}+\mathrm{H}]^{+}$215.1436, found 215.1436.

1-(4-Hexylphenyl)ethanone (3l). ${ }^{18}$ Obtained as a colorless oil (76 $\mathrm{mg}, 74 \%) .{ }^{1} \mathrm{H}$ NMR $\left(\mathrm{CDCl}_{3}, 500 \mathrm{MHz}\right): \delta 7.88(\mathrm{~d}, J=8.3 \mathrm{~Hz}, 2 \mathrm{H})$, $7.27(\mathrm{~d}, J=8.2 \mathrm{~Hz}, 2 \mathrm{H}), 2.69-2.62(\mathrm{t}, J=7.7 \mathrm{~Hz}, 2 \mathrm{H}), 2.58(\mathrm{~s}, 3 \mathrm{H})$, $1.67-1.58(\mathrm{~m}, 2 \mathrm{H}), 1.38-1.24(\mathrm{~m}, 6 \mathrm{H}), 0.92-0.82(\mathrm{~m}, 3 \mathrm{H}) .{ }^{13} \mathrm{C}\left\{{ }^{1} \mathrm{H}\right\}$ NMR $\left(\mathrm{CDCl}_{3}, 125 \mathrm{MHz}\right): \delta 197.9,148.9,135.0,128.7,128.5,36.1$, $31.8,31.2,29.0,26.6,22.7,14.2$.

1-(4-Isobutylphenyl)ethanone $(3 \mathrm{~m}) .^{19}$ Obtained as a colorless oil (69 mg, 78\%). ${ }^{1} \mathrm{H}$ NMR $\left(\mathrm{CDCl}_{3}, 500 \mathrm{MHz}\right): \delta 7.87(\mathrm{~d}, J=8.2 \mathrm{~Hz}$, $2 \mathrm{H}), 7.23(\mathrm{~d}, J=8.3 \mathrm{~Hz}, 2 \mathrm{H}), 2.58(\mathrm{~s}, 3 \mathrm{H}), 2.53(\mathrm{~d}, J=7.2 \mathrm{~Hz}, 2 \mathrm{H})$, $1.90(\mathrm{~m}, 1 \mathrm{H}), 0.91(\mathrm{~d}, J=6.6 \mathrm{~Hz}, 6 \mathrm{H}) .{ }^{13} \mathrm{C}\left\{{ }^{1} \mathrm{H}\right\} \mathrm{NMR}\left(\mathrm{CDCl}_{3}, 125\right.$ $\mathrm{MHz}): \delta$ 198.0, 147.7, 135.1, 129.4, 128.4, 45.5, 30.2, 26.6, 22.4.

1-(4-(2-(Cyclohex-3-en-1-yl)ethyl)phenyl)ethanone (3n). Obtained as a light yellow oil $(62 \mathrm{mg}, 55 \%) .{ }^{1} \mathrm{H}$ NMR $\left(\mathrm{CDCl}_{3}, 500\right.$ $\mathrm{MHz}): \delta 7.88(\mathrm{~d}, J=8.1 \mathrm{~Hz}, 2 \mathrm{H}), 7.27(\mathrm{~d}, J=8.1 \mathrm{~Hz}, 2 \mathrm{H}), 5.71-5.62$ $(\mathrm{m}, 2 \mathrm{H}), 2.71(\mathrm{t}, J=7.8 \mathrm{~Hz}, 2 \mathrm{H}), 2.58(\mathrm{~s}, 3 \mathrm{H}), 2.20-1.99(\mathrm{~m}, 3 \mathrm{H})$, $1.83-1.52(\mathrm{~m}, 5 \mathrm{H}), 1.34-1.21(\mathrm{~m}, 1 \mathrm{H}) .{ }^{13} \mathrm{C}\left\{{ }^{1} \mathrm{H}\right\} \operatorname{NMR}\left(\mathrm{CDCl}_{3}, 125\right.$ $\mathrm{MHz}): \delta 197.9,148.9,135.1,128.7,128.6,127.2,126.4,38.2,33.4$, $33.2,31.9,28.9,26.6,25.2$. IR (neat): $\nu=3021,2912,2836,1680$, $1605,1433,1412,1356,1302,1265,1181,1017,954,871,847,818$, $689,653,596,581 \mathrm{~cm}^{-1}$. HRMS (ESI): $m / z$ calcd for $\mathrm{C}_{16} \mathrm{H}_{21} \mathrm{O}[\mathrm{M}+$ $\mathrm{H}]^{+}$229.1592, found 229.1590 .

1-(4-(3-Methoxypropyl)phenyl)ethanone (30). Obtained as a colorless oil $(72 \mathrm{mg}, 75 \%) .{ }^{1} \mathrm{H}$ NMR $\left(\mathrm{CDCl}_{3}, 500 \mathrm{MHz}\right): \delta 7.89$ $(\mathrm{d}, J=8.2 \mathrm{~Hz}, 2 \mathrm{H}), 7.28(\mathrm{~d}, J=8.2 \mathrm{~Hz}, 2 \mathrm{H}), 3.38(\mathrm{t}, J=6.3 \mathrm{~Hz}, 2 \mathrm{H})$, $3.34(\mathrm{~s}, 3 \mathrm{H}), 2.75(\mathrm{t}, J=7.8 \mathrm{~Hz}, 2 \mathrm{H}), 2.58(\mathrm{~s}, 3 \mathrm{H}), 1.95-1.85(\mathrm{~m}$, $2 \mathrm{H}) .{ }^{13} \mathrm{C}\left\{{ }^{1} \mathrm{H}\right\}$ NMR $\left(\mathrm{CDCl}_{3}, 125 \mathrm{MHz}\right): \delta 197.9,147.9,135.2,128.8$, 128.6, 71.7, 58.7, 32.4, 31.0, 26.6. IR (neat): $\nu=2926,2867,1679$, $1606,1430,1412,1386,1357,1266,1207,1181,1115,1073,1017$, 955, 886, 845, 814, 597, $584 \mathrm{~cm}^{-1}$. HRMS (ESI): $\mathrm{m} / z$ calcd for $\mathrm{C}_{12} \mathrm{H}_{17} \mathrm{O}_{2}[\mathrm{M}+\mathrm{H}]^{+}$193.1229, found 193.1231.

$\mathrm{N}$-(3-(4-Acetylphenyl)propyl)-2-oxoazepane-1-carboxamide (3p). Obtained as a colorless oil $(86 \mathrm{mg}, 57 \%) .{ }^{1} \mathrm{H}$ NMR $\left(\mathrm{CDCl}_{3}, 500\right.$ $\mathrm{MHz}): \delta 9.35$ (br s, $1 \mathrm{H}), 7.88(\mathrm{~d}, J=8.3 \mathrm{~Hz}, 2 \mathrm{H}), 7.29(\mathrm{~d}, J=8.2 \mathrm{~Hz}$, $2 \mathrm{H}), 4.03-3.93(\mathrm{~m}, 2 \mathrm{H}), 3.32(\mathrm{q}, J=5.7 \mathrm{~Hz}, 2 \mathrm{H}), 2.77-2.66(\mathrm{~m}$, $4 \mathrm{H}), 2.58(\mathrm{~s}, 3 \mathrm{H}), 1.96-1.86(\mathrm{~m}, 2 \mathrm{H}), 1.81-1.67(\mathrm{~m}, 6 \mathrm{H}) .{ }^{13} \mathrm{C}\left\{{ }^{1} \mathrm{H}\right\}$ NMR $\left(\mathrm{CDCl}_{3}, 125 \mathrm{MHz}\right): \delta 197.9,179.6,155.0,147.4,135.2$, 128.7, 128.7, 43.9, 40.0, 39.9, 33.3, 30.8, 29.2, 28.4, 26.7, 23.6. IR (neat): $\nu=$ $1695,1680,1651,1606,1526,1453,1435,1397,1358,1333,1267$, $1213,1179,1163,1082,969,844,729,645,595 \mathrm{~cm}^{-1}$. HRMS (ESI): $m / z$ calcd for $\mathrm{C}_{18} \mathrm{H}_{25} \mathrm{~N}_{2} \mathrm{O}_{3}[\mathrm{M}+\mathrm{H}]^{+} 317.1865$, found 317.1877.

1-Cyclohexylnaphthalene (3q). ${ }^{20}$ Obtained as a yellow semisolid $(69 \mathrm{mg}, 63 \%) .{ }^{1} \mathrm{H}$ NMR $\left(\mathrm{CDCl}_{3}, 500 \mathrm{MHz}\right): 8.11(\mathrm{~d}, J=8.5,1 \mathrm{H})$, $7.84(\mathrm{~d}, J=7.8,1 \mathrm{H}), 7.68(\mathrm{~d}, J=8.0,1 \mathrm{H}), 7.52-7.35(\mathrm{~m}, 4 \mathrm{H}), 3.34-$ $3.30(\mathrm{~m}, 1 \mathrm{H}), 2.10-1.80(\mathrm{~m}, 5 \mathrm{H}), 1.75-1.49(\mathrm{~m}, 4 \mathrm{H}), 1.40-1.28(\mathrm{~m}$, $1 \mathrm{H}) .{ }^{13} \mathrm{C}\left\{{ }^{1} \mathrm{H}\right\}$ NMR $\left(\mathrm{CDCl}_{3}, 125 \mathrm{MHz}\right): \delta 143.9,134.0,131.5,129.0$, 126.3, 125.8, 125.7, 125.3, 123.3, 122.4, 39.4, 34.3, 27.4, 26.7.

( \pm )-1-Bicyclo[2.2.1]heptan-2-yl)naphthalene (3s). Obtained as a colorless oil $(80 \mathrm{mg}, 72 \%) .{ }^{1} \mathrm{H}$ NMR $\left(\mathrm{CDCl}_{3}, 500 \mathrm{MHz}\right): \delta 8.09$ (d, $J$ $=8.3 \mathrm{~Hz}, 1 \mathrm{H}), 7.83(\mathrm{~d}, J=8.0 \mathrm{~Hz}, 1 \mathrm{H}), 7.67(\mathrm{~d}, J=7.5 \mathrm{~Hz}, 1 \mathrm{H})$, $7.53-7.43(\mathrm{~m}, 2 \mathrm{H}), 7.43-7.34(\mathrm{~m}, 2 \mathrm{H}), 3.43-3.32(\mathrm{~m}, 1 \mathrm{H}), 2.59(\mathrm{~d}, J$ $=3.5 \mathrm{~Hz}, 1 \mathrm{H}), 2.38(\mathrm{~s}, 1 \mathrm{H}), 2.02-1.94(\mathrm{~m}, 1 \mathrm{H}), 1.79-1.58(\mathrm{~m}, 4 \mathrm{H})$, $1.57-1.22(\mathrm{~m}, 3 \mathrm{H}) .{ }^{13} \mathrm{C}\left\{{ }^{1} \mathrm{H}\right\}$ NMR $\left(\mathrm{CDCl}_{3}, 125 \mathrm{MHz}\right): \delta 143.2$, 134.2, 132.1, 128.9, 126.1, 125.6, 125.5, 125.4, 124.4, 121.7, 43.4, 41.7, 39.7, 37.1, 36.7, 30.6, 29.4. IR (neat): $\nu=2948,2868,1739,1596$, 1509, 1452, 1396, 1372, 1311, 1297, 1239, 1139, 1045, 950, 907, 793, $775,731,648,560 \mathrm{~cm}^{-1}$. HRMS (ESI): $\mathrm{m} / z$ calcd for $\mathrm{C}_{17} \mathrm{H}_{18}\left[\mathrm{M}^{+}\right]$ 222.1409 , found 222.1414 .

3-(4-(((Trifluoromethyl)sulfonyl)oxy)phenyl)propyl Acetate (3t). Obtained as a yellow oil $(167 \mathrm{mg}, 97 \%) .{ }^{1} \mathrm{H}$ NMR $\left(\mathrm{CDCl}_{3}, 500\right.$ $\mathrm{MHz}): \delta 7.26(\mathrm{~d}, J=8.6 \mathrm{~Hz}, 2 \mathrm{H}), 7.19(\mathrm{~d}, J=8.6 \mathrm{~Hz}, 2 \mathrm{H}), 4.09(\mathrm{t}, J=$ $6.4 \mathrm{~Hz}, 2 \mathrm{H}), 2.72(\mathrm{t}, J=7.6 \mathrm{~Hz}, 2 \mathrm{H}), 2.05(\mathrm{~s}, 3 \mathrm{H}), 2.00-1.92(\mathrm{~m}$, $2 \mathrm{H}) .{ }^{13} \mathrm{C}\left\{{ }^{1} \mathrm{H}\right\} \mathrm{NMR}\left(\mathrm{CDCl}_{3}, 125 \mathrm{MHz}\right): \delta 171.2,148.0,141.9,130.2$, $121.4,118.8(\mathrm{q}, J=319.5 \mathrm{~Hz}), 63.6,31.7,30.1,21.0 .{ }^{19} \mathrm{~F}$ NMR $\left(\mathrm{CDCl}_{3}, 470 \mathrm{MHz}\right):-72.9$. IR (neat): $\nu=1737,1501,1419,1367$, $1246,1205,1179,1135,1039,1017,884,846,813,734,638,606,568$, $535,517,498 \mathrm{~cm}^{-1}$. HRMS (ESI): $\mathrm{m} / z$ calcd for $\mathrm{C}_{12} \mathrm{H}_{13} \mathrm{~F}_{3} \mathrm{O}_{5} \mathrm{SNa}[\mathrm{M}$ $+\mathrm{Na}]^{+}$349.0333, found 349.0334. 


\section{ASSOCIATED CONTENT}

\section{S Supporting Information}

The Supporting Information is available free of charge on the ACS Publications website at DOI: 10.1021/acs.joc.6b00800.

Additional experimental details and spectral data (PDF)

\section{AUTHOR INFORMATION}

\section{Corresponding Author}

*E-mail: gmolandr@sas.upenn.edu.

Notes

The authors declare no competing financial interest.

\section{ACKNOWLEDGMENTS}

We thank Dr. Matthieu Jouffroy and Kingson Lin (University of Pennsylvania) for the preparation of alkylsilicates. We thank NIGMS (RO1 GM113878) for financial support of this research. We thank Evonik for their generous donation of trialkoxysilanes for the synthesis of alkylbis(catecholato) silicates.

\section{REFERENCES}

(1) Metal-Catalyzed Cross-Coupling Reactions; de Meijere, A., Diederich, F., Eds.; Wiley-VCH: Weinheim, Germany, 2004.

(2) Hartwig, J. F. Organotransition Metal Chemistry: From Bonding to Catalysis, 3rd ed.; University Science: Sausalito, CA, 2010.

(3) (a) Tellis, J. C.; Primer, D. N.; Molander, G. A. Science 2014, 345, 433. (b) Zuo, Z.; Ahneman, D. T.; Chu, L.; Terrett, J. A.; Doyle, A. G.; MacMillan, D. W. C. Science 2014, 345, 437. (c) Osawa, M.; Nagai, H.; Akita, M. Dalton Trans. 2007, 827. (d) Kalyani, D.; McMurtrey, K. B.; Neufeldt, S. R.; Sanford, M. S. J. Am. Chem. Soc. 2011, 133, 18566. (e) Ye, Y.; Sanford, M. S. J. Am. Chem. Soc. 2012, 134, 9034. (f) Rueping, M.; Koenigs, R. M.; Poscharny, K.; Fabry, D. C.; Leonori, D.; Vila, C. Chem. - Eur. J. 2012, 18, 5170. (g) Sahoo, B.; Hopkinson, M. N.; Glorius, F. J. Am. Chem. Soc. 2013, 135, 5505. (h) Shu, X. Z.; Zhang, M.; He, Y.; Frei, H.; Toste, F. D. J. Am. Chem. Soc. 2014, 136, 5844. (i) Tasker, S. Z.; Jamison, T. F. J. Am. Chem. Soc. 2015, 137, 9531.

(4) (a) Corce, V.; Chamoreau, L.-M.; Derat, E.; Goddard, J.-P.; Ollivier, C.; Fensterbank, L. Angew. Chem., Int. Ed. 2015, 54, 11414. (b) Jouffroy, M.; Primer, D. N.; Molander, G. A. J. Am. Chem. Soc. 2016, 138, 475. (c) Patel, N. R.; Kelly, C. K.; Jouffroy, M.; Molander, G. A. Org. Lett. 2016, 18, 764. (d) Jouffroy, M.; Kelly, C. K.; Molander, G. A. Org. Lett. 2016, 18, 876. (e) Leveque, C.; Chenneberg, L.; Corce, V.; Goddard, J.-P.; Ollivier, C.; Fensterbank, L. Org. Chem. Front. 2016, 3, 462.

(5) Nishigaichi, Y.; Suzuki, A.; Takuwa, A. Tetrahedron Lett. 2007, 48, 211.

(6) For the redox potential of $\left[\mathrm{Ru}(\mathrm{bpy})_{3}\right]\left(\mathrm{PF}_{6}\right)_{2}$, see: Prier, C. K.; Rankic, D. A.; MacMillan, D. W. C. Chem. Rev. 2013, 113, 5322.

(7) (a) Primer, D. N.; Karakaya, I.; Tellis, J. C.; Molander, G. A. J. Am. Chem. Soc. 2015, 137, 2195. (b) Karakaya, I.; Primer, D. N.; Molander, G. A. Org. Lett. 2015, 17, 3294. (c) El Khatib, M.; Serafim, R. A. M.; Molander, G. A. Angew. Chem., Int. Ed. 2016, 55, 254.

(8) For reviews, see: (a) Rosen, B. M.; Quasdorf, K. W.; Wilson, D. A.; Zhang, N.; Resmerita, A.; Garg, N. K.; Percec, V. Chem. Rev. 2011, 111, 1346. (b) Mesganaw, T.; Garg, N. K. Org. Process Res. Dev. 2013, 17, 29. (c) Tasker, S. Z.; Standley, E. A.; Jamison, T. F. Nature 2014, 509, 299.

(9) For select examples, see: (a) Furstner, A.; Leitner, A. Angew. Chem., Int. Ed. 2002, 41, 609. (b) Joshi-Pangu, A.; Wang, C.-Y.; Biscoe, M. R. J. Am. Chem. Soc. 2011, 133, 8478. (c) Agrawal, T.; Cook, S. P. Org. Lett. 2013, 15, 96. (d) Chen, X.; Quan, Z.-J.; Wang, X.-C. Appl. Organomet. Chem. 2015, 29, 296. (e) Doucet, H. Eur. J. Org. Chem. 2008, 2008, 2013.
(10) (a) Jutand, A.; Mosleh, A. Organometallics 1995, 14, 1810. (b) Jutand, A.; Mosleh, A. J. Org. Chem. 1997, 62, 261. (c) AlcazarRoman, L. M.; Hartwig, J. F. Organometallics 2002, 21, 491.

(11) For a detailed computational study of photoredox/nickel dual catalysis utilizing aryl halides, see: Gutierrez, O.; Tellis, J. C.; Primer, D. N.; Molander, G. A.; Kozlowski, M. C. J. Am. Chem. Soc. 2015, 137, 4896.

(12) Despite extensive studies using alkyltrifluoroborates as radical precursors in photoredox/nickel dual catalysis, the use of such starting materials has yet to prove fruitful for coupling to aryl sulfonate esters.

(13) Control studies showed that light, a Ru photocatalyst, and Ni as well as ligand catalysts were essential for reaction success; without these components, trace conversion to a cross-coupled product was observed.

(14) Note that both diisopropylammonium and triethylammonium counterions on alkylbis(catecholato)silicates show no difference in reactivity or yield.

(15) Li, X.; Wang, W.; Zhang, C. Adv. Synth. Catal. 2009, 351, 2342.

(16) Kim, D. W.; Hong, D. J.; Seo, J. W.; Kim, H. S.; Kim, H. K.; Song, C. E.; Chi, D. Y. J. Org. Chem. 2004, 69, 3186.

(17) Guo, X.; Li, C. Org. Lett. 2011, 13, 4977.

(18) Shen, Z.; Goh, K. K. K.; Yang, Y.; Lai, Y.; Wong, C. H. A.; Cheong, H.; Loh, T. Angew. Chem., Int. Ed. 2011, 50, 511.

(19) Bellale, E. V.; Bhalerao, D. S.; Akamanchi, K. G. J. Org. Chem. 2008, 73, 9473

(20) Czaplik, W. M.; Mayer, M.; von Wangelin, A. J. Angew. Chem., Int. Ed. 2009, 48, 607. 